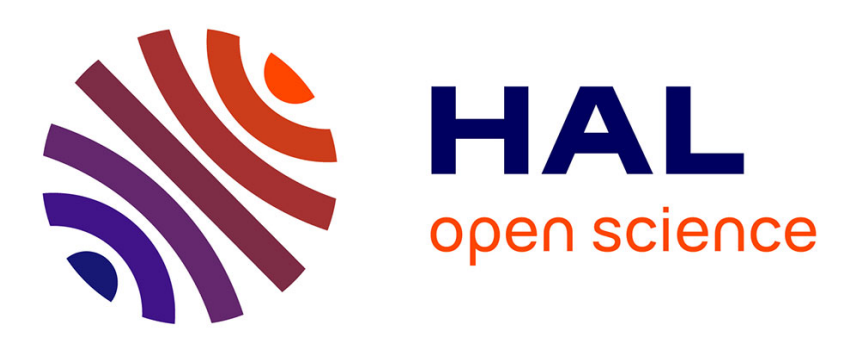

\title{
Solar Wind Charge Exchange Emission in the Chandra Deep Field North
}

Jonathan D. Slavin, Bradford J. Wargelin, Dimitra Koutroumpa

\section{To cite this version:}

Jonathan D. Slavin, Bradford J. Wargelin, Dimitra Koutroumpa. Solar Wind Charge Exchange Emission in the Chandra Deep Field North. The Astrophysical Journal, 2013, 779 (1), pp.13. 10.1088/0004-637X/779/1/13 . hal-00913265

\section{HAL Id: hal-00913265 \\ https://hal.science/hal-00913265}

Submitted on 25 Aug 2020

HAL is a multi-disciplinary open access archive for the deposit and dissemination of scientific research documents, whether they are published or not. The documents may come from teaching and research institutions in France or abroad, or from public or private research centers.
L'archive ouverte pluridisciplinaire HAL, est destinée au dépôt et à la diffusion de documents scientifiques de niveau recherche, publiés ou non, émanant des établissements d'enseignement et de recherche français ou étrangers, des laboratoires publics ou privés. 


\title{
SOLAR WIND CHARGE EXCHANGE EMISSION IN THE CHANDRA DEEP FIELD NORTH
}

\author{
Jonathan D. Slavin ${ }^{1}$, Bradford J. Wargelin ${ }^{1}$, and Dimitra Koutroumpa ${ }^{2}$ \\ ${ }^{1}$ Harvard-Smithsonian Center for Astrophysics, 60 Garden Street, Cambridge, MA 02138, USA \\ ${ }^{2}$ LATMOS/IPSL, CNRS, Université Versailles Saint Quentin, 11 Boulevard d'Alembert, F-78280, Guyancourt, France \\ Received 2013 June 28; accepted 2013 October 24; published 2013 November 18
}

\begin{abstract}
The diffuse soft X-ray background comes from distant galaxies, from hot Galactic gas, and from within the solar system. The latter emission arises from charge exchange between highly charged solar wind ions and neutral gas. This so-called solar wind charge exchange (SWCX) emission is spatially and temporally variable and interferes with our measurements of more distant cosmic emission while also providing important information on the nature of the solar wind-interstellar medium interaction. We present the results of our analysis of eight Chandra observations of the Chandra Deep Field North (CDFN) with the goal of measuring the cosmic and SWCX contributions to the X-ray background. Our modeling of both geocoronal and heliospheric SWCX emission is the most detailed for any observation to date. After allowing for $\sim 30 \%$ uncertainty in the SWCX emission and subtracting it from the observational data, we estimate that the flux of cosmic background for the CDFN in the $\mathrm{O}$ vII $\mathrm{K} \alpha$, $\mathrm{K} \beta$, and O VIII Ly $\alpha$ lines totals $5.8 \pm 1.1$ photons s $\mathrm{sm}^{-2} \mathrm{sr}^{-1}$ (or LU). Heliospheric SWCX emission varied for each observation due to differences in solar wind conditions and the line of sight through the solar system, but was typically about half as strong as the cosmic background (i.e., one-third of the total) in those lines. The modeled geocoronal emission was $0.82 \mathrm{LU}$ in one observation but averaged only $0.15 \mathrm{LU}$ in the others. Our measurement of the cosmic background is lower than but marginally consistent with previous estimates based on XMM-Newton data.
\end{abstract}

Key words: Sun: heliosphere - X-rays: diffuse background - X-rays: ISM

Online-only material: color figures

\section{INTRODUCTION}

The diffuse soft X-ray background (SXRB) has long been thought to manifest the role of massive stars in shaping the local interstellar medium (LISM). The existence of the large ( 50-200 pc) extremely low density region that surrounds us, known as the Local Bubble or Cavity (e.g., Welsh et al. 2010), bolsters the claim that supernovae and/or stellar winds have energized the LISM. The combination of the Local Bubble and the SXRB was used to create a simple model for the LISM known as the displacement model (Snowden et al. 1990) in which the cavity is filled with uniform-emissivity hot gas $\left(T \approx 10^{6} \mathrm{~K}\right.$ ) that produces the SXRB, with spatial variations in brightness caused by differences in path length to the bubble edge. In this picture, higher density neutral gas has been displaced from the Local Bubble region to the bubble walls by the processes that created the bubble.

The interior of the Local Bubble is not entirely devoid of higher density gas, however. Within a distance of $\sim 15 \mathrm{pc}$ from the Sun, 15 clouds have been identified that are generally low density $\left(n \sim 0.2 \mathrm{~cm}^{-3}\right)$, partially ionized $(\sim 20 \%-30 \%$ for $\mathrm{H}$; Slavin \& Frisch 2008), and warm ( $T \sim 7000 \mathrm{~K}$; Redfield \& Linsky 2008). The solar system itself resides in one such cloud as evidenced by, among other things, inflowing interstellar neutrals $(\mathrm{H}$ and $\mathrm{He}$ ) that have been directly detected near the Sun (Witte 2004; Möbius et al. 2004; Bzowski et al. 2008). The interaction of the solar wind with the local interstellar cloud (LIC), which has a velocity relative to the Sun of $\sim 23.5 \mathrm{~km} \mathrm{~s}^{-1}$ (Möbius et al. 2012; Bzowski et al. 2012), creates the heliosphere. Ions in the LIC are prevented from entering the heliosphere by the magnetic field carried outward by the solar wind. The neutrals, however, have mean free paths that are large compared with the heliosphere, which extends more than $100 \mathrm{AU}$ from the Sun $(\sim 90$ AU to the termination shock in the upwind direction), and follow roughly ballistic trajectories through the solar system unless ionized by solar-UV photoionization, electron impacts, or charge exchange (a.k.a. charge transfer). This interaction of interstellar neutrals with highly ionized solar wind ions, in addition to removing neutrals from the interstellar inflow, produces emission that has a profound impact on the interpretation of SXRB observations.

The solar wind is dominated by protons but includes ionized helium $(\sim 2 \%-5 \%$ depending on wind speed and solar cycle phase; Aellig et al. 2001) and a small fraction $(\sim 0.2 \%)$ of highly ionized heavier elements. Oxygen is the most abundant of the "metals" and is mostly in the form of $\mathrm{O}^{6+}$ and $\mathrm{O}^{7+}$, often with a small fraction of $\mathrm{O}^{8+}$. When a highly charged ion undergoes charge exchange with neutral $\mathrm{H}$ or $\mathrm{He}$ the resulting ion is left in an excited state and the ensuing cascade to the ground state generates a range of emission lines. Much of this solar wind charge exchange (SWCX) emission is in soft X-rays and mimics the emission from hot gas at the energy resolution of the detectors used to map out the diffuse SXRB (e.g., Koutroumpa et al. 2009). Models of this SWCX require as inputs the speed and density of the solar wind ions and the interstellar neutrals over the line of sight (LOS) through the heliosphere. SWCX emission also arises in the tenuous outer atmosphere of Earth. This exospheric, or geocoronal, emission is typically an order of magnitude weaker than heliospheric emission because of its smaller column density of neutral gas.

The photon scattering cross section and mass of neutral $\mathrm{H}$ are such that interstellar $\mathrm{H}$ atoms are somewhat deflected as they approach the Sun. The $\mathrm{H}$ also suffers significant ionization near the Sun and thus SWCX emission from heliospheric $\mathrm{H}$ peaks in the upwind direction, with severe depletion of neutral $\mathrm{H}$ in the downwind direction. In contrast, neutral He is focused by 

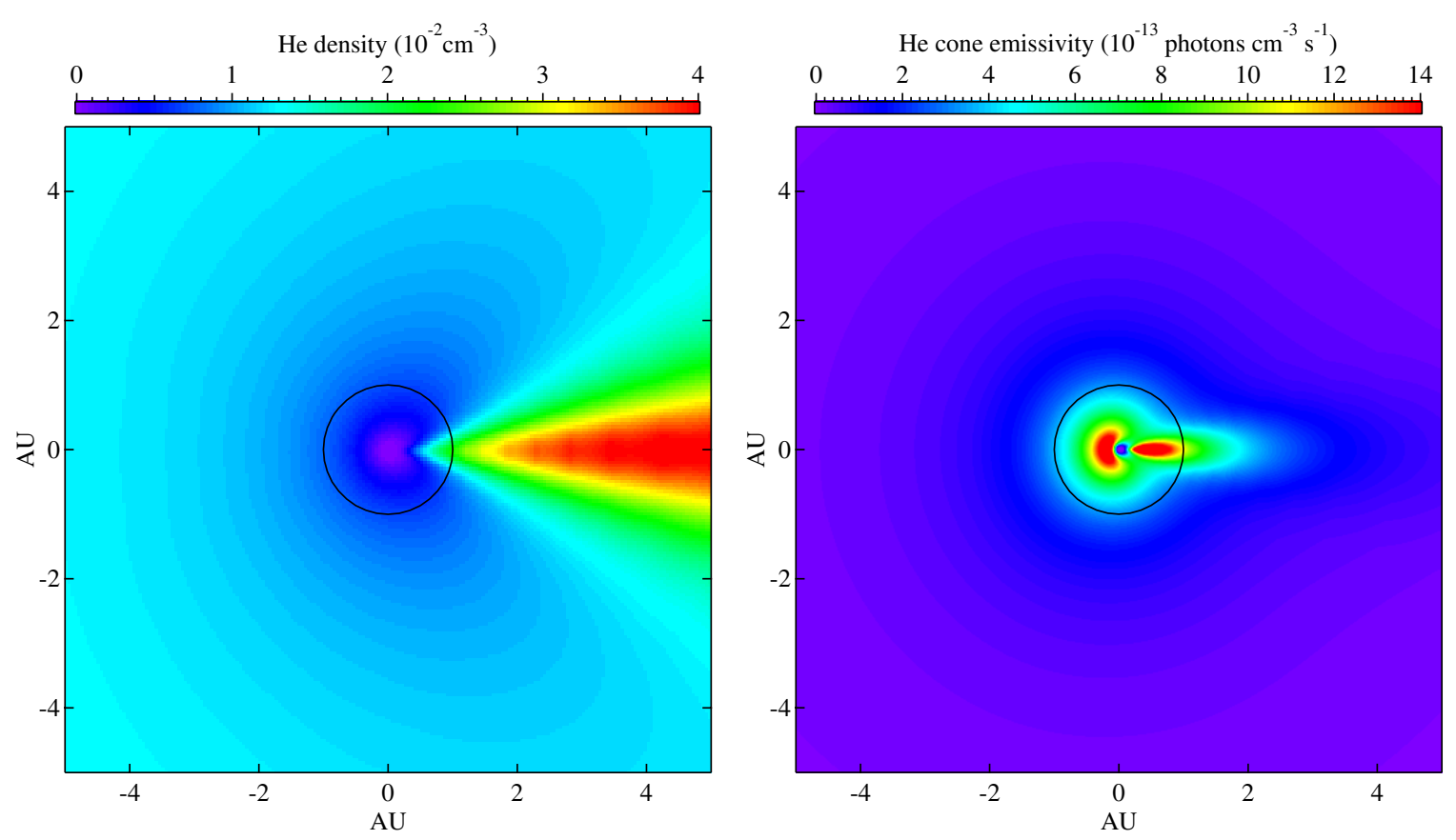

Figure 1. Modeled He density (left) and emissivity of the O vII $0.57 \mathrm{keV}$ triplet due to SWCX with $\mathrm{He}$ atoms (right) as seen from the North ecliptic pole during solar maximum. Interstellar neutrals move from the left to the right. The black circle shows Earth's orbit.

(A color version of this figure is available in the online journal.)

the gravitational force of the Sun resulting in a substantially enhanced region of SWCX emission on the downwind side of the Sun known as the He focusing cone (see Figure 1).

The solar wind is highly variable in both space and time but we have only incomplete information on its characteristics at any given point, with continuous high quality coverage only available for the near-Earth region. As discussed by Geiss et al. (1995), the solar wind has two main components: a slow $\left(\sim 400 \mathrm{~km} \mathrm{~s}^{-1}\right)$ highly ionized wind, and a fast, less ionized, lower density wind. During solar minimum these two components are mostly separated, with the slow wind confined within $10^{\circ}$ or $20^{\circ}$ of the ecliptic. During solar maximum, when our observations took place, the two components mix at most latitudes with the slow component usually dominating. The density of neutral heliospheric gas is also somewhat reduced during solar maximum. As first explained by Cravens et al. (2001), most of the observed SWCX emission originates within a few or several AU of Earth, and variations in the solar wind cause corresponding variations in the observed heliospheric emission on time scales ranging from hours to days or weeks. Variations on time scales as short as tens of minutes are also seen and come from geocoronal emission, which arises within a few tens of Earth radii and therefore closely reflects local solar wind behavior.

The Chandra $X$-ray Observatory is an excellent instrument for high resolution X-ray spectroscopy and photometry. Although not specifically designed to detect low surface brightness diffuse emission, its high spatial resolution permits the removal of point sources down to very low levels, and the low and well characterized background of the ACIS CCD detectors can then be accurately subtracted. In this paper, we report on our study of diffuse soft X-ray emission in the Chandra Deep Field North (CDFN), focusing on variations in the observed emission - the sum of extragalactic, Galactic, and local emission-and comparing with detailed models of heliospheric and geocoronal SWCX emission.

\section{CHANDRA DEEP FIELD NORTH DATA}

The CDFN, which encompasses the Hubble Deep Field North, is centered on $(\alpha, \delta)=\left(12^{\mathrm{h}} 37^{\mathrm{m}},+62^{\circ} 14^{\prime}\right)$ and was observed 17 times by the ACIS-I 4-CCD detector array during 2000-2002 for a total of 1.85 million seconds. Nine of the observations were done in FAINT mode and eight in VFAINT mode, the latter for a total of $984 \mathrm{ks}$. Since in this work we are aiming to detect the faint and diffuse SWCX emission and VFAINT mode permits better removal of the cosmic ray background, we analyze only the VFAINT observations. Table 1 lists the ObsID, pointing, and exposure time (both raw and cleaned) for each of those observations.

There are two sets of VFAINT observations, one taken 2001 November 13-21 and the other 2002 February 12-22. The two sets were taken at different roll angles, the first using a roll of 40.5 (44.3 for ObsID 3389) and the second with a roll of

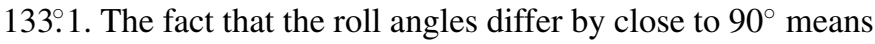
that nearly the same region of sky was observed by all eight observations. We reprocessed all of the ObsIDs with VFAINT filtering using version 4.1 of the Chandra Interactive Analysis of Observations (CIAO) software package (Fruscione et al. 2006) provided by the Chandra X-ray Center (CXC) and following the procedures outlined in the reprocessing threads on the $\mathrm{CXC}$ web pages.

One fortunate aspect of these two sets of CDFN observations for our present purposes is the orientation of the lines of sight relative to the helium focusing cone. As mentioned in the Introduction, the helium focusing cone forms on the downwind side of the Sun, where by "wind" we mean the flow of interstellar matter relative to the solar system. The gravitation of the Sun diverts the interstellar neutral He creating a density enhancement in the direction $(\lambda, \beta) \approx(79.0,-4.9)$. In Figure 1 we plot a model representation of the He density in the focusing cone and the resultant SWCX emissivity from a "hot model" (Lallement et al. 2004a, 2004b) heliosphere calculation of the trajectory 

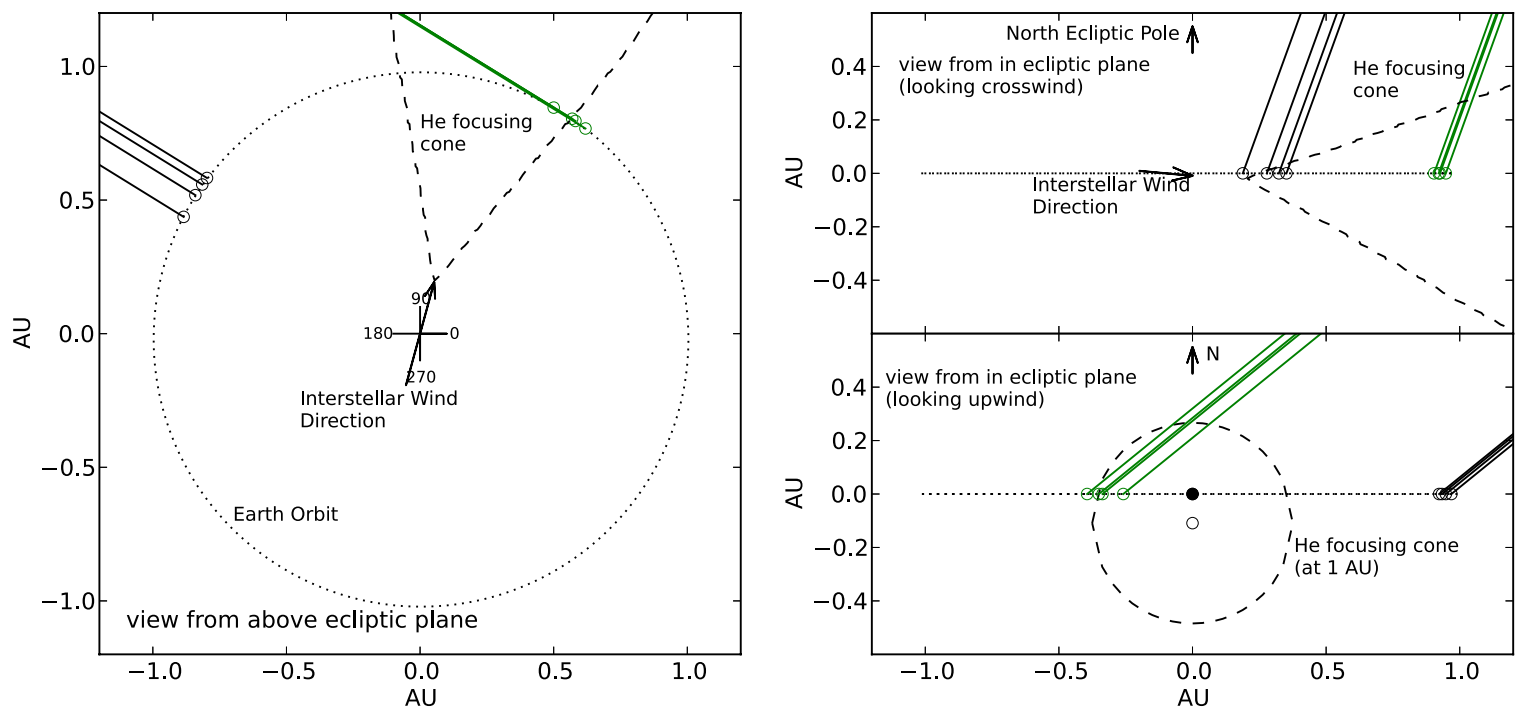

Figure 2. Illustration of the lines of sight for the two sets of CDFN VFAINT mode observations. Views from above the solar system looking down (left) and from within the ecliptic plane (right) are shown. In the left panel, ecliptic longitude $\lambda$ is shown in the diagram center. Ecliptic coordinates of the CDFN are $148.4,+57: 3$. Observations carried out in 2001 November, shown in green, can be seen to cross the helium focusing cone while the 2002 February observations do not.

(A color version of this figure is available in the online journal.)

Table 1

Chandra Deep Field North Observation Data

\begin{tabular}{llcccc}
\hline \hline ObsID & $\begin{array}{c}\text { Observation Start } \\
\text { Date }\end{array}$ & $\begin{array}{c}\text { Total Exposure } \\
(\mathrm{ks})\end{array}$ & $\begin{array}{c}\text { Cleaned Exposure } \\
(\mathrm{ks})\end{array}$ & R.A. & Decl. \\
\hline 3293 & 2001 Nov 13 & 161.3 & $73.5^{\mathrm{a}}$ & 123651.71 & +621303.10 \\
3388 & 2001 Nov 16 & 49.6 & 39.3 & 123651.71 & +621303.09 \\
3408 & 2001 Nov 17 & 66.2 & 66.2 & 123651.71 & +621303.09 \\
3389 & 2001 Nov 21 & 125.1 & $61.4^{\mathrm{b}}$ & 123651.66 & +621304.14 \\
3409 & 2002 Feb 12 & 170.4 & 82.2 & 123636.88 & +621441.78 \\
3294 & 2002 Feb 14 & 164.4 & 142.4 & 123636.88 & +621441.75 \\
3390 & 2002 Feb 16 & 164.7 & 134.6 & 123636.89 & +621441.75 \\
3391 & 2002 Feb 22 & 152.8 & 123636.88 & +621441.75 \\
\hline
\end{tabular}

Notes.

${ }^{a}$ The reduction in the exposure for ObsID 3293 is not part of the standard flare cleaning process. It is because of an observed rise in the $0.5-0.7 \mathrm{keV}$ flux coupled with the absence of $A C E$ data in the latter part of the observation (see the text).

${ }^{b}$ After running $1 c_{-}$clean this observation was judged to still have significant flare contamination and was not included in further analysis.

and ionization of interstellar neutral He in the heliosphere. This image is for a plane containing the initial inflow vector (with $\beta \approx-5^{\circ}$ ); the density displayed is therefore very close to that expected in the plane of Earth's orbit. In Figure 2 we illustrate the lines of sight toward the CDFN as viewed looking down on the ecliptic plane, along with views in the ecliptic plane looking upwind and crosswind.

\section{SOURCE REMOVAL AND FLARE CLEANING}

Hickox \& Markevitch (2006, hereafter HM06) previously examined the CDFN observations with the aim of removing all detectable point sources in the field and presenting an absolute measurement of the unresolved $0.5-8 \mathrm{keV}$ cosmic $\mathrm{X}$-ray background. After attempting their own source detection, HM06 chose to use the source catalog from Alexander et al. (2003), which they found to be nearly identical to their own. We use the same source catalog in this work. HM06 were primarily concerned with the cosmic X-ray background above 1 or $2 \mathrm{keV}$ and used very conservative (large) exclusion regions around each source. They also completely excluded events more than $5^{\prime}$ from the aimpoint (out of the full $17^{\prime} \times 17^{\prime}$ field of view) .
We are focused on lower energy emission which is significantly brighter, and most of the point sources in the field are distant and therefore absorbed. The Chandra point-spread function (PSF) is also tighter at lower energies, so source contamination is even less of a problem. To increase the available SXRB signal we therefore relaxed the exclusion criteria of HM06, using the full field of view as our starting point and then removing sources with exclusion regions that are one third as large as theirs.

We tested the effect of using smaller source regions by repeating the source exclusion procedure using HM06-sized regions and then comparing spectra. The only (very small) discrepancy that we find is at the low energy end, $E \lesssim 0.4 \mathrm{keV}$, where HM06-region spectra have relatively more flux. We believe this occurs because most of the extra area that we gain when using smaller source regions is near the ACIS readouts, which are along the outer edges of the field where the PSF is larger. Spectra using the larger exclusion regions therefore have relatively more events from high rows of the CCDs (near the center of the field) which suffer more charge transfer inefficiency, leading to spurious low energy tails that appear as low energy events. We confirmed this by examining 


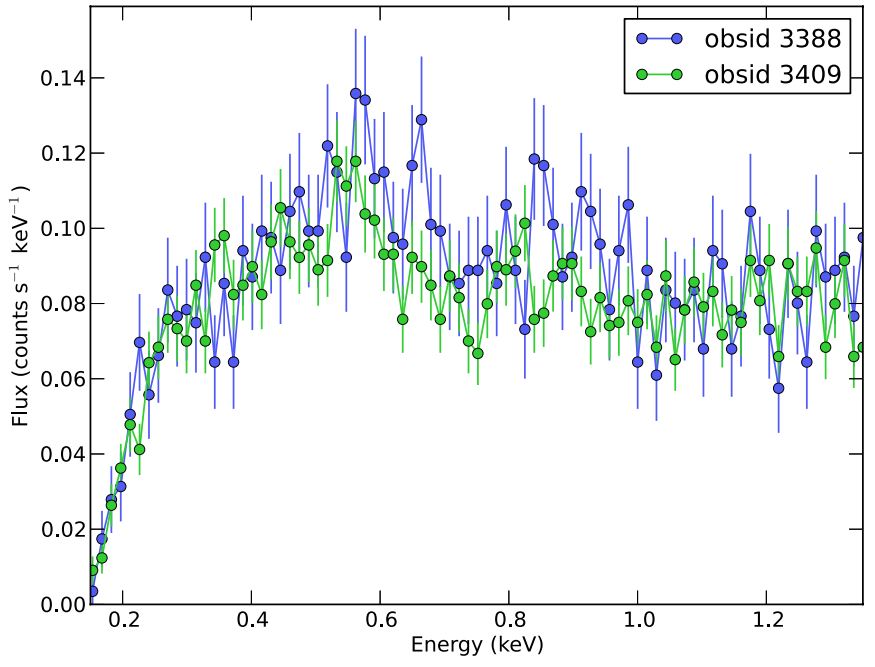

Figure 3. Comparison of SXRB spectra from ObsIDs 3388 and 3409, before subtraction of detector background. ObsID 3388, observed during 2001 November with its line of sight passing through the helium focusing cone, clearly shows excess flux over that for ObsID 3409, observed in 2002 February.

(A color version of this figure is available in the online journal.)

source-subtracted images in detector coordinates as a function of energy. In any case, ACIS sensitivity and calibration reliability both decline rapidly toward low energies so we do not include data below $0.4 \mathrm{keV}$ in our spectral fits.

Occasionally the detector background will "flare" because of an increased flux of high-energy particles, mostly from the Sun. HM06 detailed their procedures for identifying and removing such flares, and we follow very similar filtering procedures using the $1 c_{-}$clean CIAO routine; our resulting Good Time Intervals are nearly identical with theirs. Most of our observations are fairly clean (see Table 1), but ObsID 3389 shows especially large and persistent flaring with a high count rate at the beginning of the observation and again at the end, and even the middle of the light curve is not entirely flat. The spectrum also shows excess continuum emission relative to the spectra of the other ObsIDs, especially at energies below $1 \mathrm{keV}$. For this reason we have excluded ObsID 3389 from further analysis. Another special case is ObsID 3293 which, while generally free from background flaring, shows a small but significant rise in $0.5-0.7 \mathrm{keV}$ flux in the latter third of the 1.87 day observation, unlike the other six observations which have statistically constant light curves. Neither $A C E$ nor Wind solar-wind data, which would help us to diagnose the source of the rate increase, are available during this time and so we truncate this ObsID where the $A C E$ data drop out, 1.05 days before the observation ends.

\section{INSTRUMENTAL BACKGROUND SUBTRACTION}

After removing the point sources, the main source of counts is instrumental background. This background has been well characterized by the "ACIS undercover" observations that have been carried out periodically over the course of the Chandra mission and collect data while ACIS is stowed out of the focal plane of the instrument. The background's spectral shape is nearly constant with time but its rate varies by more than a factor of two over the solar cycle. The instrumental background file used was acis_D_0123567_stowed_evt_280807.fits, compiled from data collected 2000-2005. The background was reprojected onto the sky for each ObsID using the

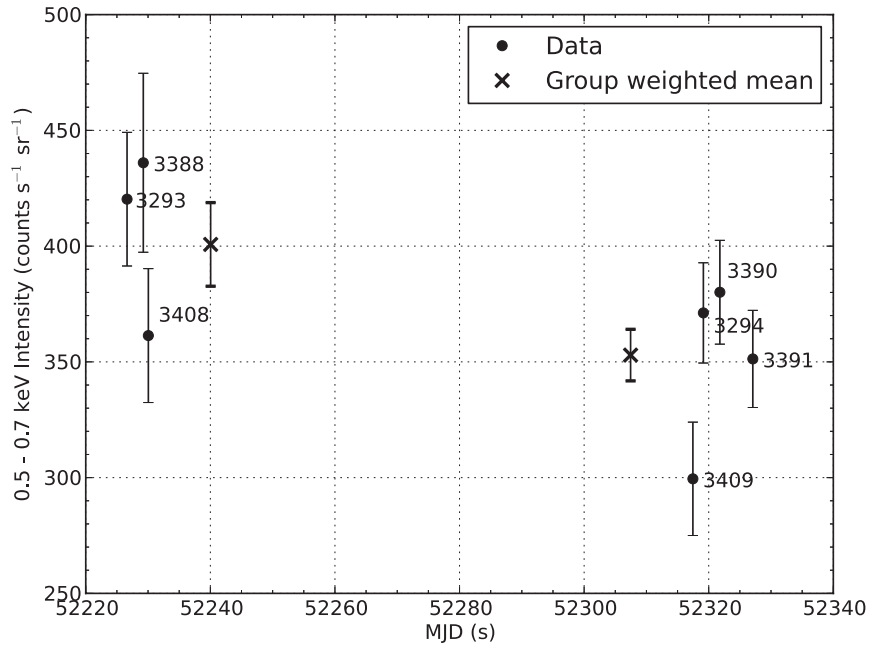

Figure 4. Intensity at $0.5-0.7 \mathrm{keV}$ after subtracting instrumental background for the seven VFAINT CDFN ObsIDs, and including a 3\% adjustment to account for lower ACIS detection efficiency in 2002 February. The weighted averages for each set of observations are also shown. The 2001 November observations have a significantly higher average intensity than the 2002 February observations, caused by different levels of SWCX emission.

CIAO routine reproject_events and the associated source regions were then excluded to account for spatial variations across ACIS. We then applied VFAINT filtering and extracted the spectrum using dmextract. These steps are explained in more detail on the CIAO Web site (http://cxc.harvard.edu/ciao/threads/acisbackground/). As expected, we find that after appropriate scaling the instrumental background created in this way is a very good match to the CDFN spectra at high energies where the Chandra effective area decreases toward zero.

The most prominent non-instrumental features in our spectra are the oxygen lines around 560 and $650 \mathrm{eV}$, as shown in Figure 3. That figure compares spectra from ObsIDs 3388 (from 2001 November) and 3409 (2002 February), showing that the former spectrum has more low energy emission. Concentrating on the 500-700 eV energy range containing the oxygen lines, we find that the average background-subtracted event rate for the three 2001 November observations is $400 \pm 18$ counts $\mathrm{s}^{-1}$, versus an average of $353 \pm 12$ counts $\mathrm{s}^{-1}$ for the four 2002 February observations (see Figure 4). Because the cosmic (Galactic and extragalactic) SXRB is constant, the observed difference must be due to different levels of SWCX emission. In the next section we present a more detailed analysis of each observation's spectrum.

\section{SPECTRAL FITTING}

Our approach to spectral fitting is similar to that of Snowden et al. (2004, hereafter SCK) in their analysis of XMMNewton observations of the CDFN. We fit our spectra with multiple components including unabsorbed thermal emission (Local Bubble), absorbed thermal emission (so-called "TransAbsorption Emission," or TAE), an absorbed extragalactic power law (presumably mostly from active galactic nuclei), and SWCX emission. Following SCK we fix the power law index to be 1.46 and the $\mathrm{H}$ I absorption column density to the Galactic value of $1.5 \times 10^{20} \mathrm{~cm}^{-2}$. The unabsorbed local thermal emission has a temperature of $k T \sim 0.09 \mathrm{keV}$ while the TAE component, presumably from the Galactic halo, has a higher temperature of $k T \sim 0.20 \mathrm{keV}$. We do not fix the temperatures, but 


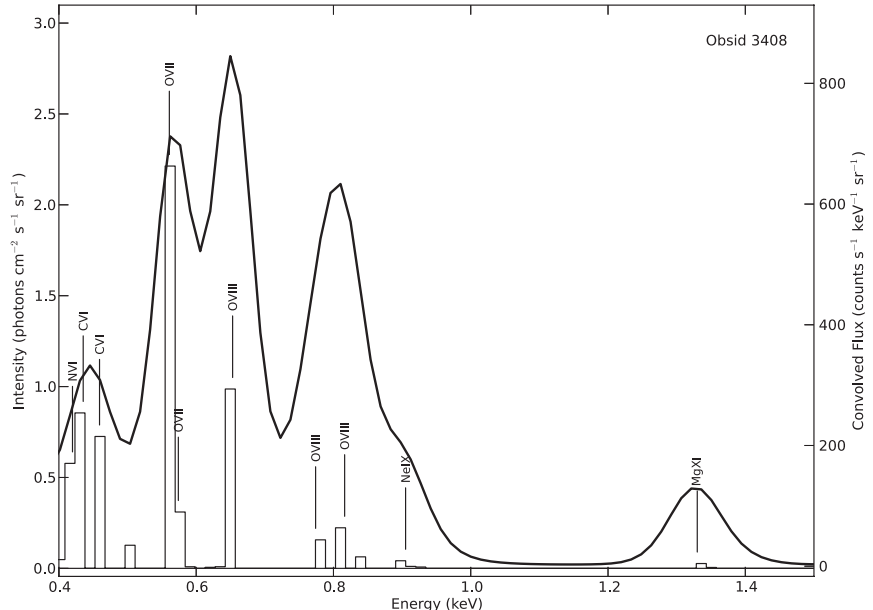

Figure 5. Modeled heliospheric SWCX spectrum using average slow-wind element abundances and ion fractions for C, N, and O taken from Schwadron \& Cravens (2000). Abundances of $\mathrm{Ne}^{9+}$ and $\mathrm{Mg}^{11+}$ are poorly determined; we use relatively large values of $\mathrm{Ne}^{9+} / \mathrm{O}=0.003$ and $\mathrm{Mg}^{11+} / \mathrm{O}=0.0015$ for illustrative purposes. This example uses the neutral gas distribution along the line of sight for ObsID 3408. The histogram (left axis scale) shows the unconvolved spectrum with labels identifying the emitting ion. The smooth curve (right axis) shows the spectrum after convolution with the ACIS response.

starting with these values leads to good fits with final values close to those listed. Unlike SCK, we do not include a second hotter TAE component since we get good fits without it and the statistics of the data do not warrant it.

To fit the spectra we use CIAO's modeling and fitting package, Sherpa (Doe et al. 2007). For thermal emission we use the XSPEC variable-abundance APEC xsvapec model ${ }^{3}$ with the phabs model ${ }^{4}$ for photoelectric absorption by the

3 XSPEC is an older but widely used X-ray spectral fitting program (see http://heasarc.nasa.gov/xanadu/xspec/ and Arnaud 1996); Sherpa models based on XSPEC equivalents are called "xspec models." APEC (Astrophysics Plasma Emission Code) is a plasma modeling code, part of the ATOMDB package (http://www.atomdb.org/), used to model X-ray spectra. We use the xsvapec model's default elemental abundances, from Anders \& Grevesse (1989).

4 The xsphabs model uses photoabsorption cross sections and element abundances from Balucinska-Church \& McCammon (1992).

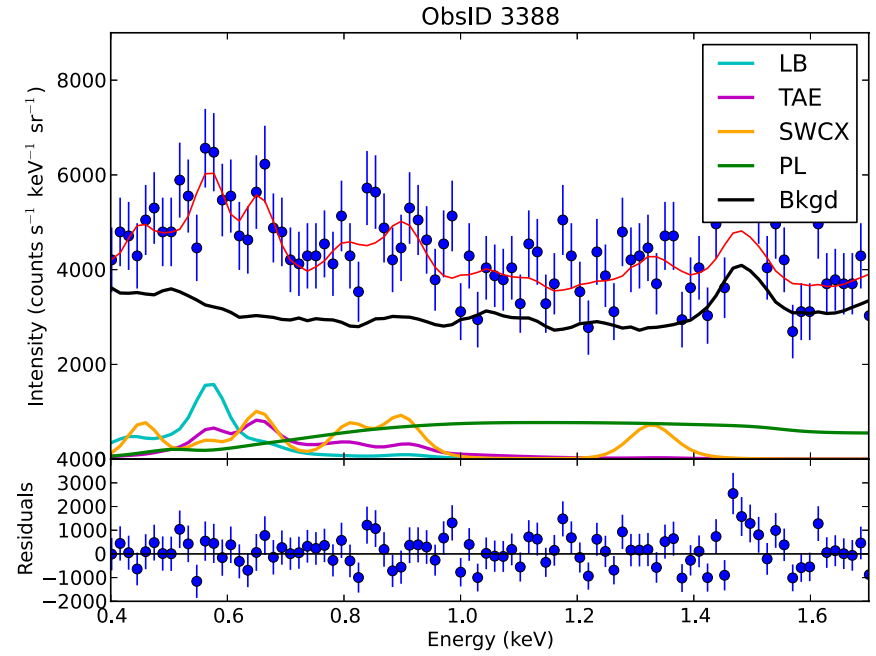

interstellar medium. We also follow SCK in fitting the strongest expected SWCX emission lines, modeled as narrow (no thermal broadening) Gaussians: C VI Ly $\gamma(459 \mathrm{eV}), \mathrm{O}$ vII $\mathrm{K} \alpha(561 \mathrm{eV})$, $\mathrm{O}$ VIII Ly $\alpha(653 \mathrm{eV}$; this model line also includes emission from $\mathrm{O}$ VII K $\beta$ at $664 \mathrm{eV}), \mathrm{O}$ VIII Ly $\gamma(816 \mathrm{eV})$, Ne Ix K $\alpha(905 \mathrm{eV})$, and $\mathrm{Mg}$ XI $\mathrm{K} \alpha(1.33 \mathrm{keV})$. These lines are shown in the example model SWCX spectrum of Figure 5.

We did the fits two different ways: with standard solar abundances, and with the oxygen abundance in the hot gas (Local Bubble and TAE) set to zero. The first approach attempts to distinguish between thermal and SWCX line emission, while the latter approach, although physically unrealistic, gives us a more accurate measure of the total emission in the $\mathrm{O}$ lines, a quantity that can vary from one observation to another only because of variations in SWCX emission. Examples of the fits for ObsIDs 3388 and 3409 are shown in Figure 6 for the solar $\mathrm{O}$ abundance case and Figure 7 for the zero $\mathrm{O}$ abundance case.

For both fitting schemes we smoothed the instrumental background using a Savitzky-Golay filter and then rescaled it for each ObsID according to the number of events at 9-12 keV, where the observed spectrum is purely instrumental. The background normalizations were then allowed to vary during the fits but only changed by a few percent from the initial values. In order to improve the fitting statistics we fit all the observations simultaneously using linked parameters for the cosmic emission (which is the same in each observation) but allowed the SWCX line intensities to vary for each spectrum. The fitted energy range was $0.4-9 \mathrm{keV}$ and the extragalactic power law was well constrained by the spectrum above $\sim 1 \mathrm{keV}$, where there is little emission from other model components.

\section{RESULTS}

Our simultaneous fits yield statistically good fits for all seven ObsIDs with reduced $\chi^{2}$ below 1.0. The results for the fitting of the distant (cosmic, non-SWCX) emission are listed in Table 2 and for the SWCX lines in Tables 3 and 4, with line brightness in units of photons $\mathrm{s}^{-1} \mathrm{~cm}^{-2} \mathrm{sr}^{-1}$, or Line Units (LU). The listed uncertainties are the $68.3 \%$ confidence limits on the parameters as calculated by the Sherpa script conf. These confidence

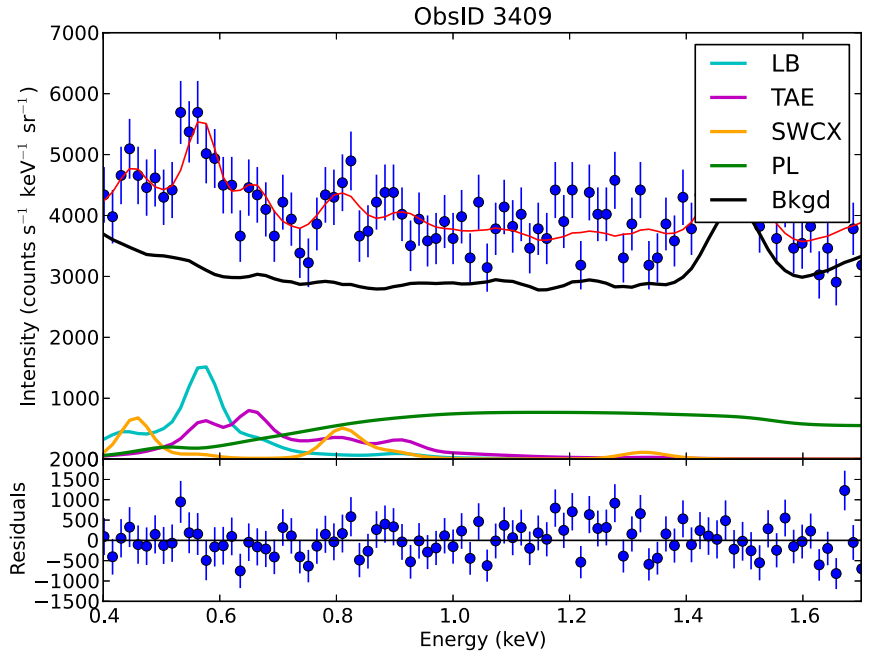

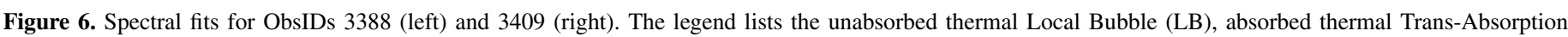

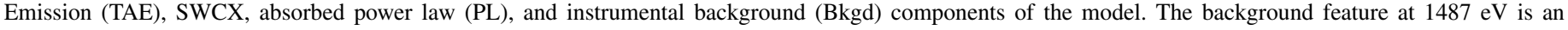

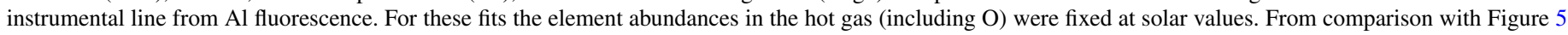
we see that emission from C VI (459 eV), O VIII $(653 \mathrm{eV})$, and $\mathrm{Mg}$ XI (1330 eV) was unusually strong during ObsID 3388.

(A color version of this figure is available in the online journal.) 

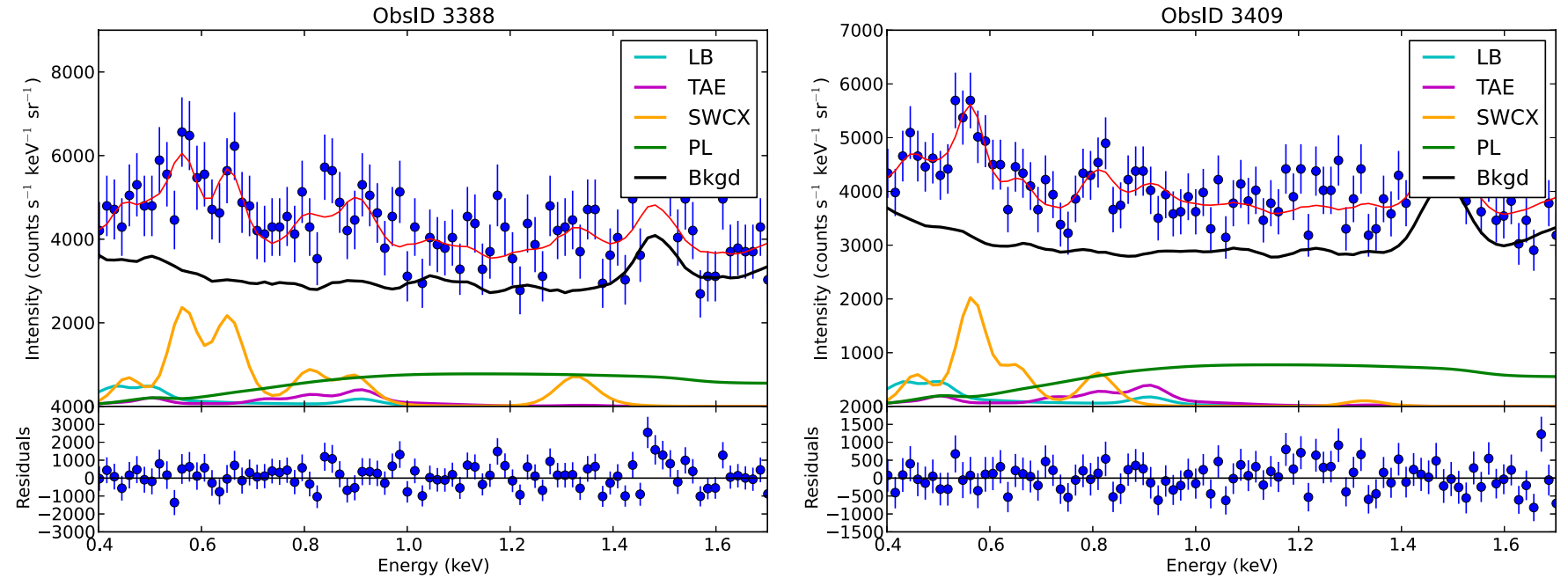

Figure 7. Same as Figure 6 except the $\mathrm{O}$ abundance in the hot gas (LB and TAE) was set to zero; the "SWCX" curve therefore denotes the total intensity $(\mathrm{LB}+\mathrm{TAE}+\mathrm{SWCX})$ in the $\mathrm{O}$ lines.

(A color version of this figure is available in the online journal.)

Table 2

Fitted Distant Emission Parameters ${ }^{\mathrm{a}}$

\begin{tabular}{lccccr}
\hline \hline Model & $\begin{array}{c}T_{\mathrm{LB}} \\
(\mathrm{keV})\end{array}$ & $\begin{array}{c}E M_{\mathrm{LB}} \\
\left(\mathrm{cm}^{-6} \mathrm{pc}\right)\end{array}$ & $\begin{array}{c}T_{\mathrm{TAE}} \\
(\mathrm{keV})\end{array}$ & $\begin{array}{c}E M_{\mathrm{TAE}} \\
\left(\mathrm{cm}^{-6} \mathrm{pc}\right)\end{array}$ & $\mathrm{PL}_{\text {norm }}{ }^{\mathrm{b}}$ \\
\hline Solar & $0.104_{-0.023}^{+0.008}$ & $0.0093_{-0.0020}^{+0.0077}$ & $0.199_{-0.055}^{+0.024}$ & $0.0012_{-0.0012}^{+0.0004}$ & $2.60_{-0.11}^{+0.16}$ \\
No O & $0.117_{-0.010}^{+0.019}$ & $0.0094_{-0.0019}^{+0.0046}$ & $0.186_{-0.072}^{+0.056}$ & $0.0024_{-0.0024}^{+0.0009}$ & $2.62_{-0.11}^{+0.17}$ \\
\hline
\end{tabular}

Notes.

${ }^{\text {a }}$ Uncertainties were calculated using the Sherpa command conf for $68.27 \%$ confidence limits. Note that for some parameters the confidence limit includes a value of zero.

${ }^{\mathrm{b}}$ Units are photons $\mathrm{keV}^{-1} \mathrm{~cm}^{-2} \mathrm{~s}^{-1} \mathrm{sr}^{-1}$ normalized at an energy of $1 \mathrm{keV}$.

Table 3

Fitted SWCX Line Fluxes for Solar O Abundance Model (in LU)

\begin{tabular}{ccccccc}
\hline \hline ObsID & \multicolumn{5}{c}{ Line Energy (eV) } \\
\cline { 2 - 7 } & 459 & 561 & 653 & 816 & 905 & 1330 \\
\hline 3293 & $3.48_{-1.43}^{+1.58}$ & $\ldots$ & $1.28_{-0.35}^{+0.86}$ & $0.34_{-0.15}^{+0.22}$ & $0.24_{-0.12}^{+0.16}$ & $0.17_{-0.07}^{+0.07}$ \\
3388 & $3.19_{-2.13}^{+2.41}$ & $1.21_{-1.21}^{+2.97}$ & $1.16_{-0.54}^{+0.90}$ & $0.37_{-0.23}^{+0.29}$ & $0.34_{-0.18}^{+0.20}$ & $0.17_{-0.10}^{+0.10}$ \\
3408 & $0.43_{-0.43}^{+1.58}$ & $\ldots$ & $0.81_{-0.37}^{+0.86}$ & $0.41_{-0.16}^{+0.23}$ & $0.12_{-0.12}^{+0.17}$ & $0.03_{-0.03}^{+0.07}$ \\
3409 & $3.04_{-1.43}^{+1.64}$ & $0.23_{-0.23}^{+2.51}$ & $\ldots$ & $0.27_{-0.15}^{+0.21}$ & $0.04_{-0.04}^{+0.14}$ & $0.03_{-0.03}^{+0.06}$ \\
3294 & $1.42_{-1.03}^{+1.27}$ & $1.43_{-0.84}^{+2.92}$ & $\ldots$ & $0.09_{-0.09}^{+0.19}$ & $\ldots$ & $0.04_{-0.04}^{+0.05}$ \\
3390 & $3.40_{-1.09}^{+1.32}$ & $\ldots$ & $0.72_{-0.24}^{+0.82}$ & $0.07_{-0.07}^{+0.20}$ & $0.03_{-0.03}^{+0.12}$ & $0.03_{-0.03}^{+0.05}$ \\
3391 & $2.24_{-1.01}^{+1.25}$ & $0.10_{-0.10}^{+2.83}$ & $0.13_{-0.13}^{+0.78}$ & $0.22_{-0.10}^{+0.20}$ & $0.10_{-0.07}^{+0.11}$ & $0.05_{-0.04}^{+0.04}$ \\
& & & & & &
\end{tabular}

Note. ${ }^{\mathrm{a}} \mathrm{LU}=$ Line Unit $=$ photons $\mathrm{s}^{-1} \mathrm{~cm}^{-2} \mathrm{sr}^{-1}$.

limits are one-dimensional projections of the multidimensional confidence region. That is, they are the regions that include $68.3 \%$ of the probability surrounding the best fit value for each parameter with all the other parameters allowed to vary in order to minimize the $\chi^{2}$. The method used is described in more detail at http://cxc.cfa.harvard.edu/sherpa/ahelp/conf.html.

Remarkably, fits for both hot-gas abundance assumptions (solar for all, and zero oxygen but otherwise solar) lead to emission measures and temperatures for the hot gas components that are nearly identical (see Table 2). As can be seen in Figure 7, the Local Bubble emission for the case of zero $\mathrm{O}$ abundance is significant only in the $\sim 0.4-0.5 \mathrm{keV}$ range, while the TAE emission is important only near $0.9 \mathrm{keV}$. As Table 2 indicates,
Table 4

Fitted SWCX Line Fluxes for $A_{\mathrm{O}}=0 \operatorname{Model}($ in LU)

\begin{tabular}{ccccccc}
\hline \hline ObsID & \multicolumn{5}{c}{ Line Energy (eV) } \\
\cline { 2 - 7 } & 459 & $561^{\mathrm{a}}$ & $653^{\mathrm{a}}$ & $816^{\mathrm{a}}$ & 905 & 1330 \\
\hline 3293 & $2.88_{-1.40}^{+1.65}$ & $6.20_{-1.17}^{+1.41}$ & $2.56_{-0.40}^{+0.44}$ & $0.40_{-0.15}^{+0.24}$ & $0.16_{-0.10}^{+0.21}$ & $0.17_{-0.07}^{+0.07}$ \\
3388 & $2.56_{-2.04}^{+2.24}$ & $8.21_{-1.87}^{+2.05}$ & $2.41_{-0.58}^{+0.63}$ & $0.43_{-0.22}^{+0.30}$ & $0.26_{-0.15}^{+0.28}$ & $0.17_{-0.09}^{+0.09}$ \\
3408 & $\ldots$ & $5.95_{-1.24}^{+1.48}$ & $2.09_{-0.41}^{+0.45}$ & $0.47_{-0.16}^{+0.24}$ & $0.05_{-0.05}^{+0.21}$ & $0.03_{-0.03}^{+0.06}$ \\
3409 & $2.37_{-1.42}^{+1.68}$ & $7.50_{-1.17}^{+1.41}$ & $0.79_{-0.35}^{+0.39}$ & $0.33_{-0.13}^{+0.23}$ & $\ldots$ & $0.03_{-0.03}^{+0.06}$ \\
3294 & $0.78_{-0.78}^{+1.36}$ & $8.55_{-0.88}^{+1.13}$ & $1.16_{-0.26}^{+0.31}$ & $0.13_{-0.11}^{+0.17}$ & $\ldots$ & $0.04_{-0.04}^{+0.04}$ \\
3390 & $2.76_{-1.11}^{+1.39}$ & $6.93_{-0.90}^{+1.13}$ & $1.97_{-0.28}^{+0.33}$ & $0.12_{-0.10}^{+0.20}$ & $\ldots$ & $0.03_{-0.03}^{+0.05}$ \\
3391 & $1.60_{-1.04}^{+1.32}$ & $7.13_{-0.84}^{+1.06}$ & $1.37_{-0.25}^{+0.30}$ & $0.28_{-0.09}^{+0.19}$ & $0.03_{-0.03}^{+0.16}$ & $0.05_{-0.04}^{+0.04}$ \\
\hline
\end{tabular}

Notes. ${ }^{a}$ For these fits, which set the hot-gas oxygen abundance to zero but otherwise used solar abundances, tabulated fluxes for $\mathrm{O}$ lines represent the total line emission (SWCX + LB + TAE).

the lower limit of the confidence range includes zero TAE emission for both the solar $\mathrm{O}$ abundance and zero $\mathrm{O}$ abundance cases. We note that there are a number of reasons to suspect that the Local Bubble emission is more complicated than a simple uniformly emitting collisional ionization equilibrium thermal plasma (e.g., Koutroumpa et al. 2009) and thus the emission measure derived may not be a true indication of the emission measure of the hot plasma within the Bubble. Our results nevertheless indicate the presence of substantial soft X-ray emission with little or no absorption. It is also clear from Figure 6 that although oxygen emission generally dominates SWCX spectra, 
even in the $0.5-0.7 \mathrm{keV}$ range most of the SXRB comes from non-SWCX emission (i.e., the Local Bubble, TAE, and extragalactic power law).

One approach to determining the contribution of the thermal $(\mathrm{TAE}+\mathrm{LB})$ gas to the $\mathrm{O}$ line fluxes is to subtract the modeled $\mathrm{O}$ line fluxes for the case with solar $\mathrm{O}$ abundance from those for zero $\mathrm{O}$ abundance. The difference then would be attributed to the thermal gas. Doing this yields a value of 8.0 LU of thermal emission in the $\mathrm{O}$ lines, with an average SWCX flux of 1.0 LU. It is, however, difficult to distinguish between thermal and SWCX emission with the typical $\sim 100 \mathrm{eV}$ resolution of CCD detectors, as can be seen from the large fit uncertainties, particularly for the $561 \mathrm{eV}$ line. In addition, the true $\mathrm{O}$ abundance in the thermal gas is not currently known. We therefore focus in the following section on the zero $\mathrm{O}$ abundance fits (Table 4), which give the total O line emission ( $\mathrm{LB}+\mathrm{TAE}+\mathrm{SWCX})$, and then model and subtract the SWCX emission in order to determine the level of thermal line emission.

\section{SWCX MODELING}

SWCX modeling has been discussed in many previous papers. Three of the most recent and detailed efforts to model timedependent SWCX emission are Koutroumpa et al. (2011), Robertson et al. (2012), and Carter et al. (2011). The first paper estimates heliospheric emission during an XMM-Newton observation of MBM 12, a dark cloud that blocks most of the cosmic (non-SWCX) SXRB, by extrapolating locally measured solar wind conditions outward along the LOS. Robertson et al. (2012) model geocoronal SWCX emission during an example geomagnetic storm, examining how the $\mathrm{X}$-ray emission would appear from different orbital perspectives. The last paper models geocoronal emission for nearly a hundred XMM-Newton observations that were previously identified as having significant short-term SXRB variability. Our modeling combines and extends analysis methods from these works, as described below.

\subsection{Geocoronal Charge Exchange}

Our geocoronal modeling is the most detailed effort for any X-ray observation to date. To model the solar wind's interaction with the Earth's magnetosphere we use the Block-Adaptive-Tree-Solarwind-Roe-Upwind-Scheme model (BATS-R-US; Tóth et al. 2012, 2005), version 20110131 without inner magnetosphere extensions, available via the Community Coordinated Modeling Center's Runs on Request interface at http://ccmc.gsfc.nasa.gov/requests/requests.php. This is the same magnetospheric model used by Robertson et al. (2012) and should be more accurate than the method used by Carter et al. (2011), although we apply it to far fewer observations. The BATS-R-US code solves MHD equations in three dimensions using an adaptive grid composed of rectangular blocks of varying sizes in Geocentric Solar Magnetospheric (GSM) coordinates. $^{5}$

The simulation volume extends from -250 to +33 Earth radii $\left(R_{E}\right)$ in $X$ and from -48 to $+48 R_{E}$ along $Y$ and $Z$, and we use the high-resolution grid (1,958,688 cells) with block sizes ranging from 0.25 to $8 R_{E}$. Model inputs are: solar wind proton density, bulk velocity, and temperature measured by the Advanced Composition Explorer ${ }^{6}$ (ACE ) SWEPAM instrument (McComas et al. 1998) with one minute binning and

\footnotetext{
5 The GSM $X$ axis points from Earth to the Sun and the $X Z$ plane contains the magnetic dipole axis.

6 ACE data are available at http://www.srl.caltech.edu/ACE/ASC/.
}

time-of-flight corrections to account for the position of $A C E$ roughly 0.01 AU "upwind" of Earth; and the time-dependent geomagnetic field strength approximated by a dipole.

There are many output parameters but the only ones we require are the proton density, velocity, and temperature, and a status field which specifies whether a particular simulation block is inside or outside the magnetopause or in a polar cusp region. All these parameters are reported as functions of time and position, with selectable block sizes; we chose time averaging of 20 minutes with spatial blocking of $0.5-3 R_{E}$. Status values near the cusps can be somewhat uncertain but our observations' lines of sight did not intercept those regions.

Those data and Chandra's orbital information (recorded at five-minute intervals) are read into our FORTRAN program geoCX.f, which then integrates the brightness of a geocoronal CX line $l$ emitted from ion $i$ along Chandra's LOS according to

$$
B_{i l}=\frac{1}{4 \pi} \int_{0}^{\infty} \epsilon_{i l} d x \text { photons s}{ }^{-1} \mathrm{~cm}^{-2} \mathrm{sr}^{-1},
$$

where $x$ is distance from Chandra and the position-dependent emissivity is given by

$$
\epsilon_{i l}=v_{c} n_{H} n_{i} y_{i l} \sigma_{i} \text { photons } \mathrm{s}^{-1} \mathrm{~cm}^{-3},
$$

where $v_{c}$ is the collision velocity (effectively the ion velocity), $n_{H}$ is the neutral hydrogen density of the exosphere, $n_{i}$ is the relevant ion density, $y_{i l}$ is the net line emission yield per $\mathrm{CX}$-excited ion, and $\sigma_{i}$ is the total CX cross section for ion $i$. Brightnesses are computed for He-like $\mathrm{O} \mathrm{K} \alpha$ and $\mathrm{K} \beta$ and $\mathrm{H}$-like Ly $\alpha$, with line yields $y_{i l}$ of 0.865 for $\mathrm{K} \alpha, 0.121$ for $\mathrm{K} \beta$, and 0.707 for $\mathrm{Ly} \alpha$. The cross sections $\sigma_{i}$ for $\mathrm{CX}$ with exospheric $\mathrm{H}$ are set to $3.40 \times 10^{-15} \mathrm{~cm}^{2}$ for $\mathrm{O}^{7+}$ and $5.65 \times 10^{-15} \mathrm{~cm}^{2}$ for $\mathrm{O}^{8+}$. Line yields and cross sections are taken from Wargelin et al. (2004) and references therein.

Oxygen ion bulk velocities and thermal velocities are assumed to be the same as the BATS-R-US proton values, and the thermal velocity $\left(v_{\text {therm }}=\sqrt{3 k_{b} T_{p} / m_{p}}\right)$ is added in quadrature to the bulk motion to obtain the total collision velocity $v_{c}$. Because BATS-R-US only calculates ion parameters for protons, oxygen ion density is based on proton density, multiplied by the solar wind $\mathrm{He} / \mathrm{H}$ abundance ratio (from ACE SWEPAM, with $1 \mathrm{hr}$ binning), $\mathrm{O} / \mathrm{He}$ ratio, and oxygen ion fraction (the latter two from $A C E$ SWICS/SWIMS (Gloeckler et al. 1998) with $2 \mathrm{hr} \mathrm{binning}^{7}$ ). We apply $1 \mathrm{hr}$ time-of-flight adjustments to the $A C E$ data and set the oxygen ion density to zero inside the magnetopause, where the solar wind is assumed to be excluded.

The exospheric neutral $\mathrm{H}$ density is probably the largest source of uncertainty in our calculations. Densities are usually inferred from measurements of scattered Lyman $\alpha$ photons and are difficult to determine beyond $\sim 10 R_{E}$. For comparison, the subsolar point of the magnetopause is typically $10 R_{E}$ and Chandra orbits between 3 and $20 R_{E}$. The most recent such measurements were made by the TWINS satellites in 2008 during solar minimum, as reported by Zoennchen et al. (2011). Their results indicate that night side densities are roughly twice as large as on the day side near $10 R_{E}\left(\sim 25 \mathrm{~cm}^{-3}\right.$ for the latter), with the primary density dependence factor being $r^{-2.993}$ where $r$ is geocentric radial distance. They also note that uncertainties grow rapidly beyond $\sim 8 R_{E}$, from around $30 \%$ to perhaps $100 \%$ at $10 R_{E}$. Fuselier et al. (2010) used IBEX observations

\footnotetext{
7 We use the latest SWICS/SWIMS data, version 3.3.1, from 2013 June. This version corrects a scaling error in the version $3.32 \mathrm{hr}$ He density data.
} 

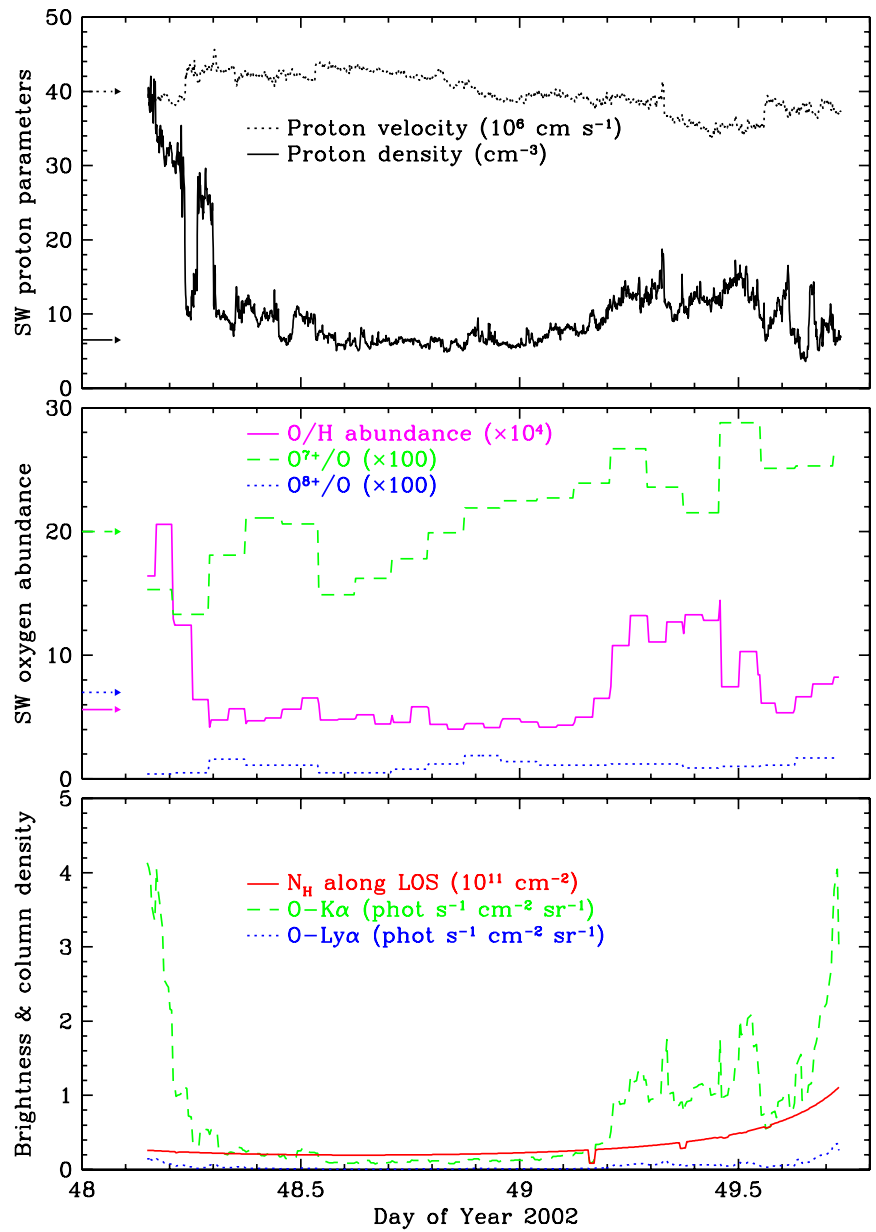

Figure 8. Model geocoronal emission for ObsID 3390. Top and middle panels show model inputs from $A C E$ (incident solar wind proton density and velocity, $\mathrm{O}$ element and ion abundances). Average values for the slow solar wind (Schwadron \& Cravens 2000) are marked on the left with arrows. Bottom panel shows the integrated neutral H column density along the Chandra LOS as it climbs and then descends in its orbit, and the predicted $\mathrm{O}$ line emission. Dips (e.g., near DoY 49.2) occur when the LOS intercepts the interior of the magnetosphere, whose boundary moves in response to the dynamic solar wind. The rise in line emission toward the end is driven both by increased oxygen ion flux and higher neutral density as Chandra's LOS passes closer to Earth.

(A color version of this figure is available in the online journal.)

of energetic neutral atoms (from charge exchange between solar wind protons and exospheric hydrogen atoms) in 2009 to estimate the exospheric density as $4-11 \mathrm{~cm}^{-3}$ at the subsolar point, with perhaps a factor of two uncertainty.

The Hodges (1994) model suggests a fairly minor dependence on solar cycle, with density at $10 R_{E}$ being perhaps $30 \%$ higher at solar minimum than at solar maximum. Given the uncertainties, that most of the SWCX emission observed by X-ray missions arises near (but outside) the magnetopause and on the day side or flanks, and that most models and measurements indicate a density of roughly $25 \mathrm{~cm}^{-3}$ at $10 R_{E}$, we adopt the simple Cravens et al. (2001) formula for exospheric atomic hydrogen density, $n_{H}=25\left(10 R_{E} / r\right)^{3} \mathrm{~cm}^{-3}$, which is an approximation to the Hodges (1994) model. Line emission is integrated out to 50 Earth radii and during the few time intervals of missing or low-quality $A C E$ data, emissivity is set to zero.

Results for ObsID 3390, which has the brightest geocoronal emission (an average combined $\mathrm{O}$ line brightness of $0.82 \mathrm{LU}$ ), are shown in Figure 8. The other six observations have an average brightness of $0.14 \mathrm{LU}$, ranging from $0.33 \mathrm{LU}$ in
ObsID 3293 down to $0.03 \mathrm{LU}$ in ObsID 3408. Compared to heliospheric emission (see next section), geocoronal CX is a minor contributor to the SXRB in the seven observations considered here, and too weak to cause statistically significant variations in the observed O-line light curve. It can be much stronger, however, during solar wind gusts or when the LOS passes near the subsolar point.

\subsection{Heliospheric Charge Exchange}

Our heliospheric SWCX models are based on self-consistent calculations of the X-ray line emission produced by $\mathrm{CX}$ collisions between heavy SW ions and interstellar neutrals flowing through the heliosphere, as extensively described in Koutroumpa et al. (2006, 2007). These simulations involve three-dimensional grids of the neutral $\mathrm{H}$ and $\mathrm{He}$ densities in the inner heliosphere (out to the termination shock at $\sim 100 \mathrm{AU}$ ), modulated by gravity, radiation pressure, and ionization processes). Line emissivities are integrated along the LOS using Equations (1) and (2), with the addition of terms for charge exchange with neutral He. Detailed information on the neutral gas distribution for different periods of the solar cycle is provided in Koutroumpa et al. (2009) based on references therein.

Figure 9 compares the modeled heliospheric $\mathrm{O} \mathrm{K} \alpha$ emission versus distance along the 2001 November and 2002 February lines of sight. The same constant solar wind flux is assumed in both cases to illustrate the impact of the neutral gas distribution along the LOS. The main point is that emission from all seven observations arises over many AU, although a substantial fraction (more than a third) of the SWCX emission for the 2001 November observations comes from well within 1 AU because the LOS intercepts the He cone (see Figure 2). The secondary bump around $7 \mathrm{AU}$ is where emission from SWCX with neutral $\mathrm{H}$ peaks along the LOS; the fall-off at larger distances is mostly from the $r^{-2}$ dependence of the wind density, while SWCX emission tends to decrease closer to the Sun because H atoms are more likely to become ionized. He is more resistant to ionization than $\mathrm{H}$, and is therefore responsible for most SWCX emission within $\sim 1$ AU of the Sun, whether or not the LOS intercepts the He cone.

A major uncertainty in our heliospheric calculations is setting the value of $n_{i}$ for $\mathrm{O}^{7+}$ and $\mathrm{O}^{8+}$ because the CDFN is at a high ecliptic latitude $(+57.3)$ while $A C E$ only samples wind near the ecliptic plane. ${ }^{8}$ Only the first several tenths of an AU along our observations' lines of sight can therefore be modeled with high confidence. As seen in Figure 9, this corresponds to a significant fraction of the total emission seen in the 2001 November observations but relatively little in 2002 February. The CCMC provides heliospheric models of solar wind proton flux with nearly complete coverage in latitude, but uncertainties in their predictions (Cohen et al. 2008) are currently too large to justify the effort required to run those models for this work.

A common approach to this problem is to use a "steady state" model, assuming constant properties for the solar wind appropriate to solar cycle phase, often with a latitude-dependent boundary between slow and fast wind during solar minimum. A slightly more sophisticated approach, used by Koutroumpa et al. (2007) when modeling an XMM-Newton observation of the $\mathrm{CDFN}$, traced the progress of a coronal mass ejection as it passed near or through the observation's LOS. In that particular example, however, the "dynamic" model did not

\footnotetext{
8 We checked for relevant data from Ulysses but it was too far from our observations' lines of sight.
} 


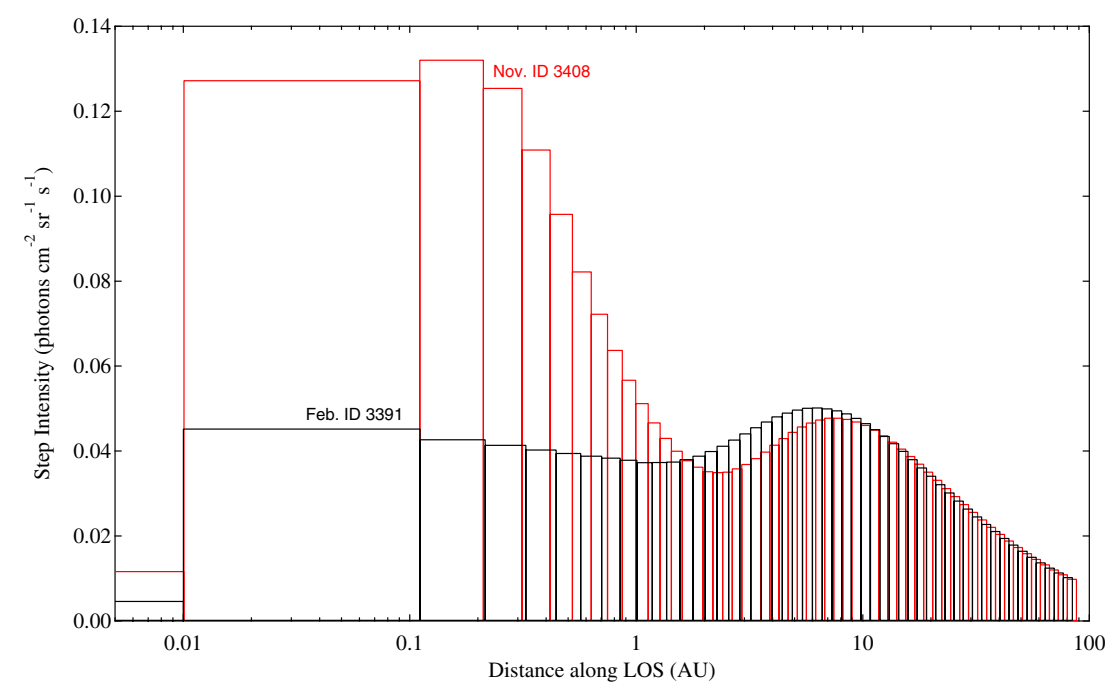

Figure 9. Model SWCX O vII K $\alpha$ flux along the LOS for representative CDFN observations from 2001 November and 2002 February, with each bin corresponding to a step of varying size along the LOS. Intensities for both curves were computed assuming average slow solar wind fluxes; the only difference is in the LOS geometry. The November observations looked through the He focusing cone while Earth was near the edge of the cone; this contributes substantial emission within 1 AU of Earth.

(A color version of this figure is available in the online journal.)

produce significantly better agreement with observation than the steady state model.

For our heliospheric modeling we use three approaches, in presumably increasing order of veracity: (1) a steady-state model, using constant solar wind parameters taken from the literature, (2) a similar method, but using average solar wind conditions measured by $A C E$ during the 30 days preceding each observation; and (3) an improved version of the dynamic model. We focus primarily on the last method but include the others for comparison.

For the steady-state model we use slow-wind oxygen ionization fractions based on Ulyssess data listed in Schwadron \& Cravens $\left(2000 ; 20 \% \mathrm{O}^{7+}\right.$ and $\left.7 \% \mathrm{O}^{8+}\right), \mathrm{O} / \mathrm{H}$ abundance ratio of $1 / 1780$, proton density of $6.5 \mathrm{~cm}^{-3}$ at $1 \mathrm{AU}$, and solar wind speed of $400 \mathrm{~km} \mathrm{~s}^{-1}$. This choice assumes that since our observations were during solar maximum, the slow wind dominated at all latitudes up to the CDFN latitude of +57.3 , and that average slow-wind parameters are constant over a solar cycle. In fact, long-term-average ionization fractions do vary somewhat over time in both the fast and slow wind (von Steiger \& Zurbuchen 2011; Schwadron et al. 2011). As a check we therefore examined $A C E$ data from 2000 and 2001 and found oxygen ion fractions of $20.6 \%$ for $\mathrm{O}^{7+}$ and $5.8 \%$ for $\mathrm{O}^{8+}$ using only "good" data (quality $=0$ or 1 ), or $19.3 \%$ and $2.7 \%$ if one includes all the data and assumes that abundances during "bad" data times (which are usually when the ion fraction is too low to measure) are zero. We therefore consider $20 \%$ and $7 \%$ to be reasonable estimates.

The rationale for the model using 30 day averaging is that most of the emission along the LOS comes from solar wind that passed by the Earth within the month preceding the corresponding Chandra observation. Helium densities and velocities, $\mathrm{O} / \mathrm{He}$ abundances, and $\mathrm{O}$ ion fractions were taken directly from $A C E$ SWICS data, with averages computed after setting bad data values to zero.

Details of the improved dynamic method are described in Koutroumpa (2012). To summarize, during each time-bin of an observation, each parcel of solar wind along the observa- tion LOS is traced to its origin on the Sun's surface. We then assume that solar wind properties are independent of solar latitude-by necessity because $A C E$ only samples wind near the ecliptic_and "rotate" that longitudinal slice of Sun backward or forward, depending on the observing geometry, to where it would emit another parcel of solar wind that could be measured by $A C E$ near the Sun-Earth L1 point. We implicitly assume that both parcels of wind (along the LOS, and sampled by $A C E$ ) have identical properties, since wind-generating structures usually do not change much on time scales shorter than the solar rotation period (assumed to be 27 days). To compute the solar wind's times-of-flight from Sun-to-LOS and Sun-to-L1 we use the appropriate $A C E$-measured radial velocity. For each time bin, every point along the LOS is now associated with a corresponding $A C E$ measurement so that the local SWCX emissivity (with the appropriate $r^{-2}$ adjustment for solar wind density) can be calculated and then integrated along the LOS to yield the predicted SWCX brightness as a function of time. Note that unlike the geocoronal model, which necessarily derives oxygen ion densities from the BATS-R-US proton density, heliospheric oxygen densities are based on the ACE SWICS He density because this is more direct, avoiding the additional $\mathrm{He} / \mathrm{H}$ factor that is needed when scaling the proton density.

All three approaches suffer from the lack of measured solar wind conditions along most of the LOS, particularly at higher solar latitudes, but as noted above our observations were made during solar maximum when there is relatively little latitudinal variation. As seen in Figure 9, SWCX emission arises over tens of $\mathrm{AU}$, corresponding to months of solar wind travel time, so that solar wind properties tend to average out over the LOS. Large unmeasured short-term deviations from typical solar wind values could lead to significant errors in the modeled SWCX if that parcel of atypical wind intercepts the LOS at a location that contributes a disproportionate fraction of the total emission. The latter condition is true for the 2001 November observations, with extra emission from the He cone, but that portion of the LOS is close to Earth where solar wind properties are well measured by $A C E$, so modeling errors should be small. 

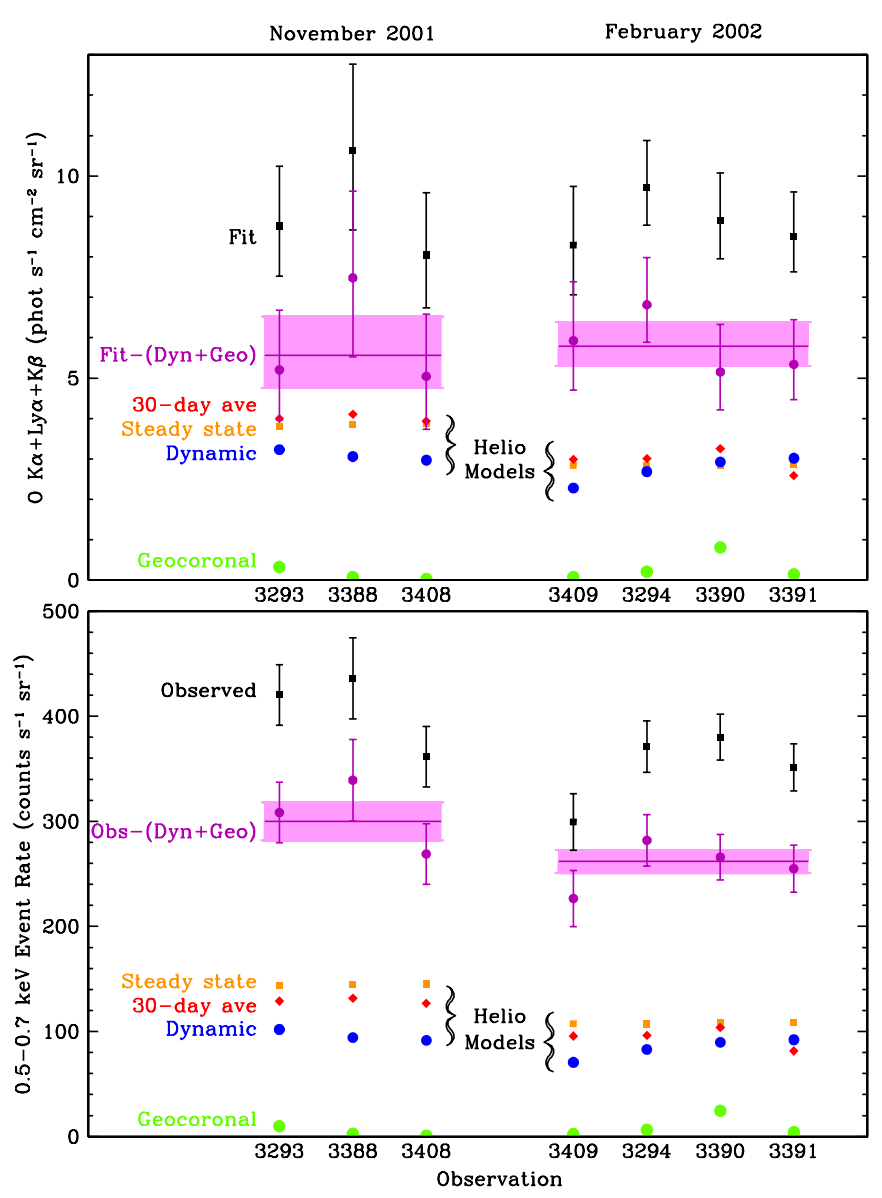

Figure 10. Observational and model results for the CDFN observations. The top panel shows total line flux from Table 4 zero $\mathrm{O}$ abundance model Fit results, and SWCX model line fluxes (from geocoronal and three heliospheric models); magenta Fit - (Dyn + Geo) points show net (cosmic) line flux using the Dynamic heliospheric model. The bottom panels show observed event rates (500-700 eV) and the rates corresponding to SWCX models in the top panel. The shaded regions denote $1 \sigma$ uncertainties on the average cosmic X-ray background emission for each group of observations.

(A color version of this figure is available in the online journal.)

\subsection{Model Predictions versus Observation}

A comparison of observed and modeled oxygen line emission is shown in Figure 10. The top panel plots line fluxes (in LU) from the zero O abundance spectral fits (Table 4), SWCX fluxes from the geocoronal and three heliospheric models, and the net emission when the model SWCX emission (using the Dynamic heliospheric model) is subtracted from the fitted flux. Recall that the Fit flux in this case is the total oxygen line emission (SWCX + Local Bubble + Trans-Absorption Emission) so the magenta Fit - (Dynamic + Geocoronal) points denote the cosmic (nonSWCX) SXRB oxygen line flux. Horizontal magenta lines mark the weighted averages for each group of data, with the shaded bands showing the $1 \sigma$ uncertainty limits.

The bottom panel of Figure 10 plots the corresponding event rates within the $500-700 \mathrm{eV}$ band dominated by $\mathrm{O}$ line emission. The Observed rates are simply taken from ACIS data; model SWCX rates are computed from the model SWCX line fluxes, multiplied by the appropriate Chandra/ACIS effective area for each line. (Note that the 2002 February event rates have been slightly adjusted to account for time-dependent changes in effective area and ease comparison with the 2001
November rates.) The 500-700 eV range includes small contributions from other SWCX ions, such as Fe L-shell emission, but a little of the $\mathrm{O}$ line emission falls outside this range because of finite detector energy resolution, so on the whole the SWCX-model-flux to event-rate conversion should be fairly accurate.

It is immediately clear that geocoronal emission is much weaker than heliospheric SWCX emission, with the previously noted partial exception of ObsID 3390. Results for the Steady State and 30-Day-Average heliospheric models are very similar; line fluxes for the 30 day model are slightly higher (with the exception of ObsID 3391) because the combined $\mathrm{O}^{7+}$ and $\mathrm{O}^{8+}$ ion flux was higher than in the Steady State model (typically $\sim 1.3 \times$ for $\mathrm{O}^{7+}$ and $\sim 0.4 \times$ for $\mathrm{O}^{8+}$ ). The 30 day event rates, however, are slightly lower (again with the exception of ObsID 3391) because the Chandra/ACIS effective area is roughly onethird as large at $560 \mathrm{eV}$ (where the $\mathrm{O}^{7+}$ ions yield most of their SWCX emission) as at $650 \mathrm{eV}\left(\mathrm{O}^{8+}\right)$.

Somewhat surprisingly, despite using much the same $A C E$ data as the 30 day model, the Dynamic model's emission is lower than both the averaging models' (except for ObsID 3391). This is because $\mathrm{O}$ ion fluxes in the few days prior to the $\mathrm{X}$-ray observations (i.e., from wind closest to the Earth) were mostly lower than average. The SWCX emission distribution for the Dynamic model (see Figure 9) is disproportionately weighted toward nearer positions along the LOS (particularly for the 2001 November observations), so this pushes all but one of the Dynamic model predictions below those using the 30 day and Steady State averages. Because of its more accurate representation of the spatial and temporal behavior of the solar wind, we use the Dynamic model's predictions to compute the net (cosmic, non-SWCX) oxygen line fluxes in Figure 10 and in all subsequent discussions.

Average net line fluxes for 2001 and 2002 are nearly the same with 5.57 ${ }_{-0.82}^{+0.96} \mathrm{LU}$ for 2001 and 5.79 ${ }_{-0.48}^{+0.60} \mathrm{LU}$ for 2002-yielding a weighted average of $5.73_{-0.42}^{+0.51}$ LU-and individual observations are all consistent with the average. For comparison, the average model SWCX oxygen line fluxes for 2001 and 2002 are 3.23 LU (3.09 heliospheric and 0.15 geocoronal) and 3.04 LU (2.73 and 0.31 ), respectively. Note that this similarity in SWCX emission is purely coincidental; the 2001 observations had a larger column of neutral gas along the LOS than the 2002 observations (because of the He cone), but the solar wind flux was higher during the latter.

In the lower panel of Figure 10, net event rates for individual observations are consistent with their corresponding group average but the average 2001 rate $\left(300 \pm 18\right.$ counts $\left.\mathrm{s}^{-1} \mathrm{sr}^{-1}\right)$ is significantly higher than that for $2002(262 \pm 11)$. The two rates can be made to match by increasing the 2001 heliospheric SWCX emission (or reducing the 2002 emission) by 40\%; a $\sim 20 \%$ adjustment will make the $1 \sigma$ error bars just overlap.

$30 \%$ is not an unreasonable estimate for the total uncertainty in our heliospheric model. The main sources of uncertainty are from CX cross sections and line yields, measured solar wind parameters, assumptions about unmeasured solar wind conditions along the LOS, and neutral gas densities. Errors in cross sections and line yields may be $\sim 30 \%$, but any errors would affect model calculations for all observations roughly equally so this cannot explain the disagreement in estimate cosmic SXRB event rates between the 2001 and 2002 observations.

$\mathrm{O}$ ion densities are derived from $A C E$ SWICS data on $\mathrm{He}^{2+}$ density, the $\mathrm{He} / \mathrm{O}$ ratio, and the $\mathrm{O}$ charge distribution. The 
SWICS release notes ${ }^{9}$ estimate an uncertainty of $30 \%$ for most parameters. SWICS He densities tend to be $\sim 25 \%$ larger (with substantial scatter) than He densities recorded by SWEPAM. This inconsistency is well known to the $A C E$ instrument teams and a subject of active research (J. Raines 2013, private communication). Any errors in the ion density measurements we use should be mostly systematic, however, and would therefore likely affect both our 2001 and 2002 modeling in the same way.

Even if $A C E$ measurements are perfect, there is the question of how well those measurements reflect solar wind conditions along the LOS, particularly at high latitude. As explained previously, SWCX emission is spread over a large volume of space, so solar wind parameters will tend to average out. Emission from nearby may sometimes contribute a relatively large share of the total (as it does here), but local solar wind conditions are well measured so errors will be small in that case. A more important factor for the rate discrepancy may be errors in the assumed neutral gas density. In the 2001 November observations the LOS intercepted the edge of the He cone, a region of enhanced He density within a larger region of lower than average $\mathrm{H}$ density (the ionized downwind side of the Sun), whereas the 2002 February lines of sight traversed regions of more typical and probably better characterized $\mathrm{H}$ and $\mathrm{He}$ density. If there are errors in the model results, we believe it is more likely that the 2001 November heliospheric emission is too large than that the 2002 February emission is too small. We therefore slightly increase the estimated net line brightness from its average of $5.73_{-0.42}^{+0.51} \mathrm{LU}$ to $5.8 \pm 0.5 \mathrm{LU}$, where the uncertainty is from the spectral fitting.

Taking all the sources of uncertainty into account, we estimate that our heliospheric modeling error is roughly $30 \%$, or $\pm 1.0 \mathrm{LU}$ for the combined emission of oxygen $\mathrm{K} \alpha, \mathrm{K} \beta$, and $\mathrm{Ly} \alpha$. Adding that in quadrature to the fitting errors for line brightness, along with $5 \%$ uncertainty in the Chandra effective area calibration (contributing $\sim 0.4 \mathrm{LU}$ ), we obtain a final estimate for the net (cosmic) SXRB emission of $5.8 \pm 1.1 \mathrm{LU}$. For comparison, the latest and most complete analysis of the $X M M$ Newton observations of the same field (Koutroumpa et al. 2007) estimated the $\mathrm{O}$ line flux as $7.87_{-1.10}^{+0.72} \mathrm{LU}$ (and note that those estimated errors do not include SWCX model uncertainties).

\section{SUMMARY AND CONCLUSIONS}

We have analyzed the SXRB in two sets of Chandra observations of the CDFN, taken only three months apart but from different points in Earth's orbit around the Sun, with substantially different lines of sight through the heliosphere. Extremely detailed model calculations of geocoronal SWCX oxygen X-ray emission were made for each observation, along with estimates of heliospheric emission using a sophisticated dynamic model that correlates each point along an observation's line of sight with a specific location on the solar surface and a corresponding $A C E$ solar wind measurement.

After subtracting the modeled SWCX emission from fitted oxygen line emission we estimate the combined intensity of $\mathrm{O}$ $\mathrm{K} \alpha, \mathrm{K} \beta$, and $\mathrm{Ly} \alpha$ from cosmic background in the CDFN as $5.8 \pm 1.1 \mathrm{LU}$, somewhat lower but in reasonable agreement with previous XMM-Newton results. Combined geocoronal and heliospheric SWCX emission in those same lines ranged from just over $2 \mathrm{LU}$ to just under $4 \mathrm{LU}$, with an average of 3.1 LU. Geocoronal emission ranged from nearly zero to

\footnotetext{
9 http://www.srl.caltech.edu/ACE/ASC/DATA/level2/ssv3/swics_lv2_v3 release_notes.txt
}

$0.82 \mathrm{LU}$, with an average of $0.24 \mathrm{LU}$. Our analysis shows the power of having multiple observations and widely separated lines of sight through the heliosphere when attempting to separate the contributions of cosmic and SWCX emission to the SXRB. We expect that detailed magnetospheric simulations such as described here, improved heliospheric models that will become available in the near future, and nondispersive X-ray detectors with high energy resolution such as on the upcoming Astro- $H$ mission will lead to significant improvements in our understanding of SWCX, the SXRB, and the structure and composition of the LISM.

We gratefully acknowledge use of SWICS and SWEPAM data provided by the $A C E$ Science Center (http://www.srl.caltech. edu/ACE/ASC/), and magnetosphere simulation results provided by the Community Coordinated Modeling Center at Goddard Space Flight Center through their public Runs on Request system (http://ccmc.gsfc.nasa.gov). The CCMC is a multi-agency partnership between NASA, AFMC, AFOSR, AFRL, AFWA, NOAA, NSF and ONR. The SWMF/BATS-RUS Model was developed by Tamas Gombosi et al. at the Center for Space Environment Modeling, University of Michigan. We also thank Michael Juda for his code to process Chandra orbital data, Ryan Hickox for advice on removing sources, and Richard Edgar for helpful discussions on solar wind ion heating. Support for this work was provided by NASA through Chandra Award Number SP1-12001X issued by the Chandra X-ray Observatory Center (CXC), which is operated by the Smithsonian Astrophysical Observatory for and on behalf of NASA under contract NAS8-03060. B.W. was supported by NASA contract NAS8-39073 to the CXC during the course of this research.

\section{REFERENCES}

Aellig, M. R., Lazarus, A. J., \& Steinberg, J. T. 2001, GeoRL, 28, 2767 Alexander, D. M., Bauer, F. E., Brandt, W. N., et al. 2003, AJ, 126, 539 Anders, E., \& Grevesse, N. 1989, GeCoA, 53, 197

Arnaud, K. A. 1996, in ASP Conf. Ser. 101, Astronomical Data Analysis Software and Systems V, ed. G. H. Jacoby \& J. Barnes (San Francisco, CA: ASP), 17

Balucinska-Church, M., \& McCammon, D. 1992, ApJ, 400, 699

Bzowski, M., Kubiak, M. A., Mbius, E., et al. 2012, ApJS, 198, 12

Bzowski, M., Möbius, E., Tarnopolski, S., Izmodenov, V., \& Gloeckler, G. 2008, A\&A, 491, 7

Carter, J. A., Sembay, S., \& Read, A. M. 2011, A\&A, 527, A115

Cohen, O., Sokolov, I. V., Roussev, I. I., \& Gombosi, T. I. 2008, JGRA, 113, 3104

Cravens, T. E., Robertson, I. P., \& Snowden, S. L. 2001, JGR, 106, 24883

Doe, S., Nguyen, D., Stawarz, C., et al. 2007, in ASP Conf. Ser. 376, Astronomical Data Analysis Software and Systems XVI, ed. R. A. Shaw, F. Hill, \& D. J. Bell (San Francisco, CA: ASP), 543

Fruscione, A., McDowell, J. C., Allen, G. E., et al. 2006, Proc. SPIE, $6270,62701 \mathrm{~V}$

Fuselier, S. A., Funsten, H. O., Heirtzler, D., et al. 2010, GeoRL, 37, L13101

Geiss, J., Gloeckler, G., von Steiger, R., et al. 1995, Sci, 268, 1033

Gloeckler, G., Cain, J., Ipavich, F. M., et al. 1998, SSRv, 86, 497

Hickox, R. C., \& Markevitch, M. 2006, ApJ, 645, 95

Hodges, R. R., Jr. 1994, JGR, 99, 23229

Koutroumpa, D. 2012, AN, 333, 341

Koutroumpa, D., Acero, F., Lallement, R., Ballet, J., \& Kharchenko, V. 2007, A\&A, 475, 901

Koutroumpa, D., Lallement, R., Kharchenko, V., et al. 2006, A\&A, 460, 289

Koutroumpa, D., Lallement, R., Raymond, J. C., \& Kharchenko, V. 2009, ApJ, 696, 1517

Koutroumpa, D., Smith, R. K., Edgar, R. J., et al. 2011, ApJ, 726, 91

Lallement, R., Raymond, J. C., Bertaux, J.-L., et al. 2004a, A\&A, 426, 867

Lallement, R., Raymond, J. C., Vallerga, J., et al. 2004b, A\&A, 426, 875

McComas, D. J., Bame, S. J., Barker, P., et al. 1998, SSRv, 86, 563

Möbius, E., Bochsler, P., Bzowski, M., et al. 2012, ApJS, 198, 11

Möbius, E., Bzowski, M., Chalov, S., et al. 2004, A\&A, 426, 897 
Redfield, S., \& Linsky, J. L. 2008, ApJ, 673, 283

Robertson, I. P., Cravens, T. E., Sibeck, D. G., Collier, M. R., \& Kuntz, K. D. 2012, AN, 333, 309

Schwadron, N. A., \& Cravens, T. E. 2000, ApJ, 544, 558

Schwadron, N. A., Smith, C. W., Spence, H. E., et al. 2011, ApJ, 739, 9

Slavin, J. D., \& Frisch, P. C. 2008, A\&A, 491, 53

Snowden, S. L., Collier, M. R., \& Kuntz, K. D. 2004, ApJ, 610, 1182

Snowden, S. L., Cox, D. P., McCammon, D., \& Sanders, W. T. 1990, ApJ, 354,211
Tóth, G., Sokolov, I. V., Gombosi, T. I., et al. 2005, JGRA, 110, 12226

Tóth, G., van der Holst, B., Sokolov, I. V., et al. 2012, JCoPh, 231, 870

von Steiger, R., \& Zurbuchen, T. H. 2011, JGRA, 116, A01105

Wargelin, B. J., Markevitch, M., Juda, M., et al. 2004, ApJ, 607,596

Welsh, B. Y., Lallement, R., Vergely, J.-L., \& Raimond, S. 2010, A\&A, 510, A54

Witte, M. 2004, A\&A, 426, 835

Zoennchen, J. H., Bailey, J. J., Nass, U., et al. 2011, AnGeo, 29, 2211 